\title{
Intracapillary immune complexes recruit and activate slan-expressing CD16+ monocytes in human lupus nephritis
}

\author{
Florina Olaru, ${ }^{1}$ Thomas Döbel, ${ }^{1}$ Anke S. Lonsdorf,' ${ }^{1}$ Stephanie Oehrl, ${ }^{1}$ Michael Maas, ${ }^{1}$ \\ Alexander H. Enk, ${ }^{1}$ Marc Schmitz, ${ }^{2,3,4,5,6}$ Elisabeth F. Gröne, ${ }^{7}$ Hermann-J. Gröne, ${ }^{7}$ and Knut Schäkel ${ }^{1}$ \\ 'Department of Dermatology, Heidelberg University Hospital, Heidelberg, Germany. ${ }^{2}$ Institute of Immunology, Medical \\ Faculty, Technische Universität (TU) Dresden, Dresden, Germany. ${ }^{3}$ National Center for Tumor Diseases, University Hospital \\ Carl Gustav Carus, TU Dresden, Germany. ${ }^{4}$ Cerman Cancer Consortium (DKTK), Dresden, Germany. ${ }^{5}$ Cerman Cancer \\ Research Center (DKFZ), Heidelberg, Germany. ${ }^{6}$ Center for Regenerative Therapies Dresden (CRTD), Medical Faculty, TU \\ Dresden, Dresden, Germany. 'Department of Cellular and Molecular Pathology, German Cancer Research Center (DKFZ), \\ Heidelberg, Germany.
}

Lupus nephritis is a major cause of morbidity in patients with systemic lupus erythematosus. Among the different types of lupus nephritis, intracapillary immune complex (IC) deposition and accumulation of monocytes are hallmarks of lupus nephritis class III and IV. The relevance of intracapillary ICs in terms of monocyte recruitment and activation, as well as the nature and function of these monocytes are not well understood. For the early focal form of lupus nephritis (class III) we demonstrate a selective accumulation of the proinflammatory population of 6-sulfo LacNAc (slan) monocytes (slanMo), which locally expressed TNF- $\alpha$. Immobilized ICs induced a direct recruitment of slanMo from the microcirculation via interaction with Fc $\gamma$ receptor IIIA (CD16). Interestingly, intravenous immunoglobulins blocked CD16 and prevented cell recruitment. Engagement of immobilized ICs by slanMo induced the production of neutrophil-attracting chemokine CXCL2 as well as TNF- $\alpha$, which in a forward feedback loop stimulated endothelial cells to produce the slanMo-recruiting chemokine CX3CL1 (fractalkine). In conclusion, we observed that expression of CD16 equips slanMo with a unique capacity to orchestrate early IC-induced inflammatory responses in glomeruli and identified slanMo as a pathogenic proinflammatory cell type in lupus nephritis.

Authorship note: FO, TD, and ASL contributed equally to this work.

Conflict of interest: The authors have declared that no conflict of interest exists.

Submitted: July 25, 2017 Accepted: April 24, 2018 Published: June 7, 2018

Reference information: JCI Insight. 2018;3(11):e96492. https://doi.org/10.1172/jci. insight. 96492.

\section{Introduction}

A number of diseases are associated with the deposition of immune complexes (ICs) in the vascular bed, the production of antibodies against the vascular basement membrane or endothelial cells, which can lead to vasculitis or glomerulonephritis (1-3). The etiology of IC formation can be either autoimmune (systemic lupus erythematosus, Goodpasture syndrome), infectious (postinfectious glomerulonephritis), or a humoral rejection of an allogeneic transplant. In these conditions ICs are formed and can be found in the walls of capillaries of the renal glomerulus, like in lupus nephritis (4), or other locations depending on the disease type resulting in local tissue injury and deterioration of endothelial function. Further, the local deposition of ICs can lead to activation of myeloid cells through their Fc receptors (FcRs) (5-7). Depending on the FCR repertoire, monocytes, macrophages, granulocytes, and dendritic cells (DCs) execute a broad spectrum of effector functions or are modulated in their activity by the presence of ICs (8). Studies in mice proposed that activation of myeloid cells by glomerular deposits of ICs requires both FcR $\gamma$ and TLRs $(9,10)$, which initiates an inflammatory response that results in glomerulonephritis (10). In stage III and IV lupus nephritis with capillary deposition of ICs, Fc $\gamma \mathrm{RIIIA}^{+}\left(\mathrm{CD} 16^{+}\right)$monocytes were noticed and regarded as relevant to the pathogenesis of the disease (11).

Monocytes are now classified based on their gradual differences in CD14 versus CD16 expression: classical CD14 ${ }^{++} \mathrm{CD} 16^{-}$, intermediate $\mathrm{CD} 14^{+} \mathrm{CD} 16^{+}$, and nonclassical CD14-CD16 ${ }^{++}$monocytes (12). Within the population of $\mathrm{CD} 16^{+} \mathrm{LIN}^{-} \mathrm{HLA}-\mathrm{DR}{ }^{+}$leukocytes (nonclassical CD14-CD $16^{++}$monocytes) our group defined the population of 6-sulfo LacNAc-expressing monocytes (slanMo). Roughly half 
of the nonclassical monocytes stain positive for slan (Supplemental Figure 1; supplemental material available online with this article; https://doi.org/10.1172/jci.insight.96492DS1). The marker molecule slan allowed the mAb-directed purification and functional studies of these cells purified from blood and tonsils, which revealed functional characteristics of DCs (13-14). Most notably, slanMo showed a strong capacity to stimulate $\mathrm{T}$ cells and to program Th1/Th17 cells (15-17). DCs, monocytes, and macrophages are now classified based on their identified precursor cell. For slan ${ }^{+}$cells, transcriptomic studies collectively demonstrated a gene signature shared with monocytes rather than bona fide DCs (18). We therefore propose calling these cells slanMo instead of slanDCs, as already recommended by others (19). The larger population of human nonclassical monocytes is regarded as equivalent to mouse patrolling monocytes (20). These patrolling CX3CR $1^{\text {hi }} \mathrm{Ly} 6 \mathrm{C}^{\mathrm{lo}}$ monocytes are guardians of the intravascular immune surveillance that showed a prolonged contact with the endothelium and orchestrate the phagocytosis of cellular debris (21). They were seen constitutively in uninflamed glomeruli (22). Prolonged interactions with the endothelial cells were found when inflammation was artificially induced by antibodies directed against endothelial cells of the glomerular capillaries. Subsequent contact with neutrophils promoted acute neutrophil-dependent glomerular injury, suggesting the critical role of murine monocytes in inflammatory glomerular diseases (23). A similar picture with neutrophil recruitment was observed when patrolling monocytes were activated by TLR7-dependent nucleic acid danger signals (21). The presence of $\mathrm{CD}^{+} 6^{+}$monocytes in human lupus nephritis with capillary IC deposition (stage IV) correlated with endothelial CX3CL1 (fractalkine) expression, which may have contributed to their recruitment (11).

In this study, we sought the identity of the monocytic cells in human lupus nephritis and their pathogenic role, with special reference to their direct recruitment from the blood circulation in the context of immobilized ICs. We previously reported an increased frequency of slanMo in the dermis of lupus skin lesions (24). In patients with systemic lupus erythematosus, ICs circulate in blood and are deposited in different organs where they contribute to organ failure. IC deposition on the surface of the glomerular basement membrane reportedly results in damage to the podocytes by mechanisms involving the activation of the complement system leading to dysfunction of the glomerular filter (25).

In lupus nephritis, mesangial deposition of ICs is found in class I and II (of the International Society of Nephrology/Renal Pathology Society [ISN/RPS] classification) (4). Intraluminal immune aggregates are present in class III and IV (of the ISN/RPS classification) lupus nephritis (26). In reference to the different sites of IC deposition, we analyzed the in vivo distribution of slanMo in a set of human lupus nephritis tissue samples spanning different classes of the disease. We identified a preferential accumulation of slanMo in class III, while in class IV other mononuclear phagocytes outnumbered slanMo. Furthermore, we recognized the direct CD16-mediated IC engagement of slanMo as a possible mechanism for the glomerular entry and adhesion of slanMo by in vitro flow chamber experiments and in a murine model of systemically administered ICs. Therapeutic doses of intravenous immunoglobulins (IVIGs) abrogated the capture of slanMo under in vitro flow conditions. We found that slanMo within glomeruli of human lupus nephritis samples express TNF- $\alpha$, and this TNF- $\alpha$ production could be reproduced by incubating slanMo with immobilized ICs. Additionally, ICstimulated slanMo produced IL-6 and the neutrophil chemoattractant CXCL2. By treating endothelial cells with supernatants from IC-stimulated slanMo, we noticed a TNF- $\alpha$-dependent production of CX3CL1 (fractalkine) by endothelial cells. This mechanism may be relevant for disease progression by recruiting additional CX3CR1 ${ }^{+}$slanMo as well as CX3CR1-expressing T cells and NK cells. Our study identifies slanMo having a functional specialization as an important proinflammatory cell type, in early IC-mediated inflammation.

A deeper insight into the pathogenesis of lupus nephritis is essential to offer new therapeutic strategies. The findings provided by this study identify slanMo as a potential therapeutic target in human lupus nephritis.

\section{Results}

SlanMo accumulate in the kidney of lupus nephritis patients. Formation and deposition of ICs is a central pathogenic mechanism for the development of lupus nephritis (27). Deposition of ICs in kidneys leads to an influx of inflammatory cells and production of proinflammatory cytokines, which promote tissue damage (28). To investigate whether glomerular IC deposition contributes to the recruitment of slanMo, 
A
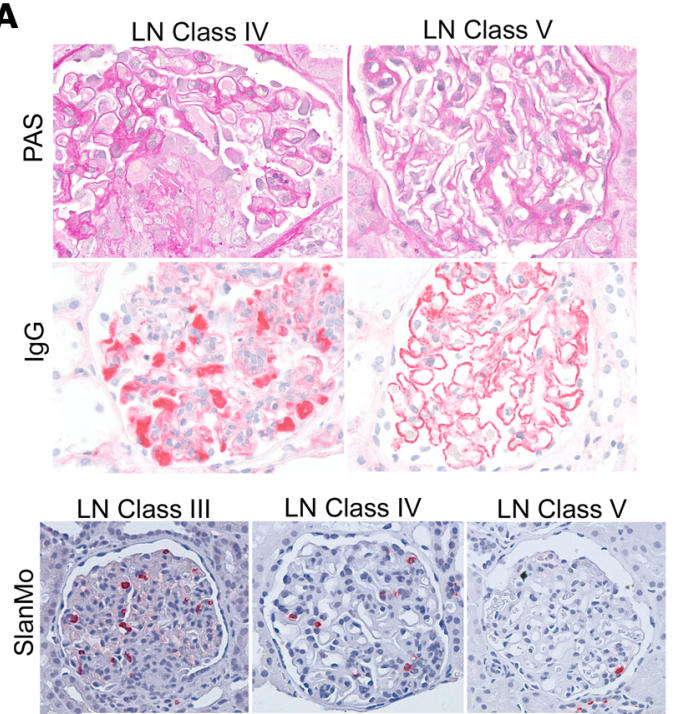

B
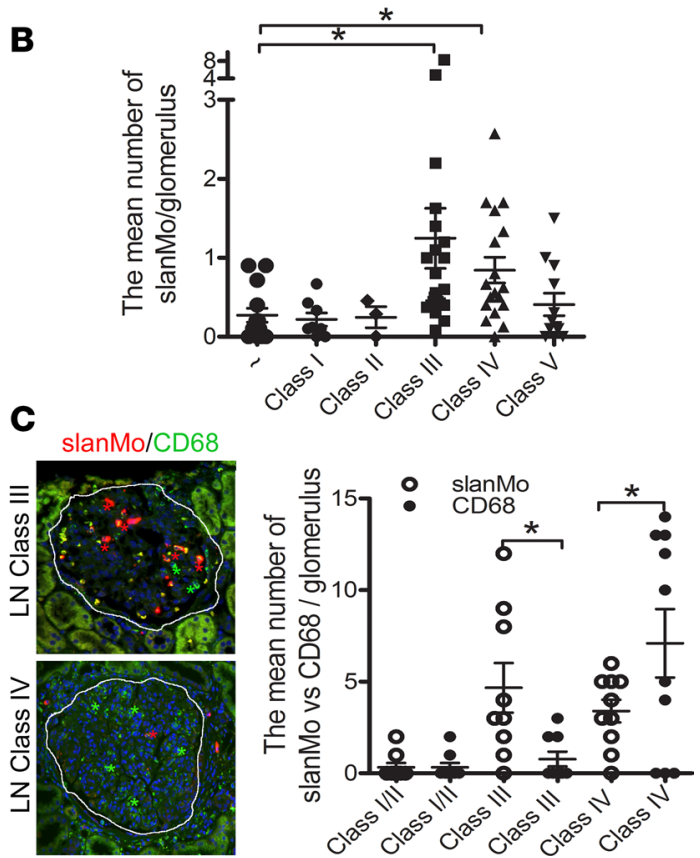

Figure 1. Preferential glomerular accumulation of slanMo in type III and IV lupus nephritis. (A) Histological images of lupus nephritis patients (class IV and V) stained with periodic acid-Schiff (PAS) and for IgG deposits and hyaline thrombi. The presence of slanMo was shown using the slan-specific antibody DD2. Original magnification, $\times 40$ (PAS and IgG) and $\times 20$ (SlanMo). (B) The mean number of immunohistochemically identified slanMo in glomeruli found in kidney samples of patients with lupus nephritis: healthy ( $n=14)$, class I $(n=8)$, class II $(n=4)$, III $(n=22)$, IV $(n=18)$ or V $(n=12)$. One-way ANOVA (nonparametric) followed by Dunn's multiple-comparison test. Mean values are shown \pm SEM. ${ }^{*} P<0.05$; comparison between healthy kidney samples and classes I-V of patients with lupus nephritis. (C) Costaining of slanMo and CD68-expressing cells (PG-M1 mAb). Original magnification, $\times 20$. The mean number of slanMo and CD68-expressing cells in glomeruli of lupus nephritis patients $(n=8-10)$. One-way ANOVA (nonparametric) followed by Newman-Keuls multiple-comparison test. Mean values are shown $\pm S E M$. ${ }^{*} P<0.05$; comparison between slanMo and CD68-expressing cells in glomeruli.

we stained kidney samples obtained from patients with lupus nephritis (class I to V of the ISN/RPS classification) for the presence of slanMo using the slanspecific antibody DD2. Classes III and IV of lupus nephritis are characterized by subendothelial and intraglomerular ICs, whereas continuous subepithelial IgG deposits in the glomerular capillary wall are a characteristic sign of class $\mathrm{V}$ membranous lupus nephritis (Figure 1A). SlanMo were chiefly identified in the glomeruli of class III and IV lupus nephritis. The mean number of slanMo per glomerulus was higher in class III and IV in comparison with the healthy controls (Figure 1B). Furthermore, double staining for CD68 and slan revealed that slanMo $\left(\mathrm{CD}^{2} 8^{+}\right)$outnumber other $\mathrm{CD} 68$-expressing cells in the glomeruli in class III lupus nephritis (Figure 1C). Interestingly, in class IV lupus nephritis we observed an infiltration of $\mathrm{CD} 68^{+}$monocytes and the majority of these cells stained negative for slan. Stage V showed no further increase in the numbers of slanMo per glomerulus but rather in the periglomerular inflammatory infiltrates (Figure 1, A and B and Supplemental Figure 2). This slanMo distribution may be correlated with the intravascular IC deposits and the presumed accessibility of subendothelial ICs by blood cells in class III and IV of lupus nephritis through the fenestrated endothelium, which is different from the subepithelial deposition of ICs in class V (26).

ICS recruit slanMo in an in vivo model. The kidney glomerulus has a fenestrated vascular bed that is thought to passively trap circulating ICs (29). We set up a mouse model to test if heat-aggregated IgG, as a surrogate of ICs, when deposited in glomeruli are able to recruit circulating human slanMo from the blood flow. A schematic representation of the experimental setting is given in Figure 2A. Mice were intravenously injected with $5 \mathrm{mg} / \mathrm{ml}$ heat-aggregated IgG followed by injection with CFSE-labeled slanMo. This reductionist approach does not reflect the complex pathology found in lupus nephritis. However, we found this model to be particularly attractive to address the Fc $\gamma$ R-dependent recruitment of cells to glomerular capillaries under in vivo conditions. To study whether the recruitment of slanMo at the site of IC deposition was CD16 mediated, corresponding experiments were performed with CD16A- or CD32A-transfected Jurkat cells. Two hours after cell injection, mice were euthanized and renal sections were analyzed by immunofluorescence for the presence of slanMo or transfected Jurkat cells in the glomeruli (Figure 2B). In this set of experiments, slanMo and CD16-transfected Jurkat cells efficiently accumulated in glomeruli of mice that had received heat-aggregated IgG. Homing of slanMo to glomeruli was largely inhibited when CD16 was blocked with specific antibodies. Similarly, untransfected WT Jurkat cells or CD32-transfected Jurkat cells did not accumulate at significant numbers in glomeruli of mice challenged with heat-aggregated IgG (Figure 2C). These experiments demonstrate that immobilized heat-aggregated IgG enables direct recruitment of cells from the flow and/or alternatively, retention of already patrolling slanMo, and identify CD16 as the critical IC receptor for this process.

ICS directly recruit slanMo in vitro. Recruitment of immune cells to inflamed tissues is guided by adhesion molecules on activated endothelium (30). To investigate whether ICs independently contribute to the 
A

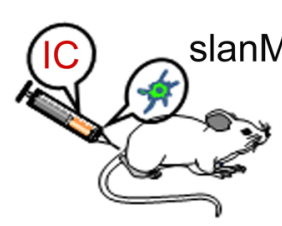

NSG mouse

B

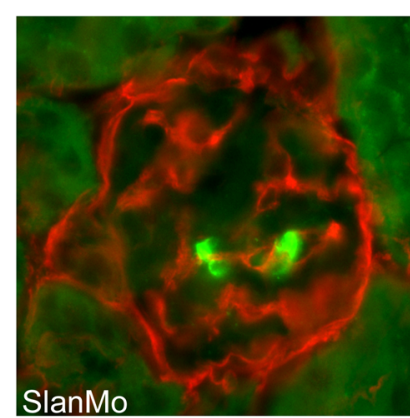

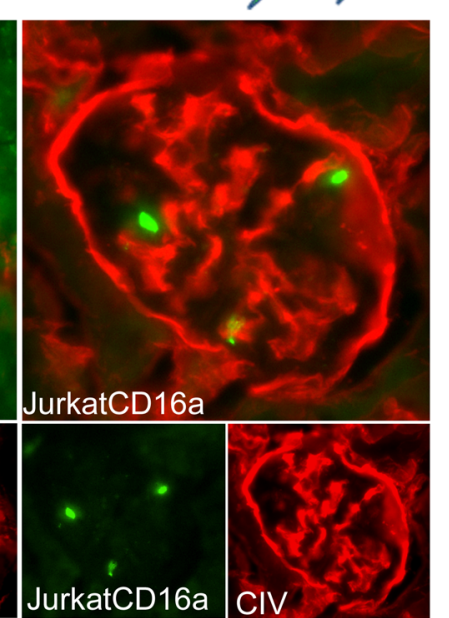

JurkatCD16a

SlanMo

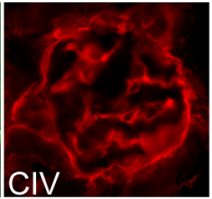

Endo

Blood
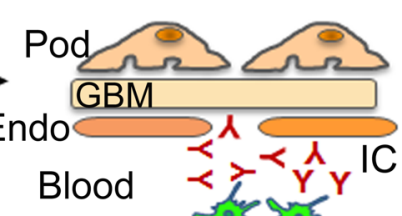

c

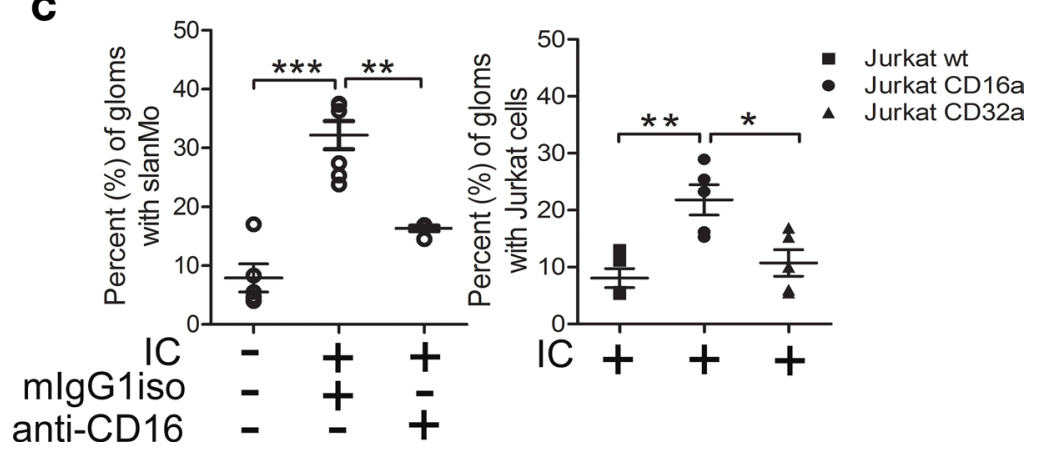

Figure 2. Recruitment of slanMo by ICs in vivo. (A) Schematic representation of the mouse model. Preformed immune complexes (ICs) were injected i.v. into immunodeficient NSG mice followed by the injection of either fluorescently labeled freshly isolated slanMo or Jurkat cells transfected with either CD16 or CD32. (B) Immunofluorescence staining of collagen IV (CIV) on kidney sections (red) of experimental animals and localization of fluorescently labeled cells (slanMo and Jurkat CD16a) (green) in the glomeruli ( $n=5$ mice per group). Original magnification, $\times 40$. (c) Percentage of glomeruli (gloms) with slanMo and Jurkat cells, respectively, recruited by ICs in the presence or absence of blocking mAbs for CD16 (3G8). Mean values are shown $\pm \operatorname{SEM}\left(n=5\right.$ mice per group). ${ }^{*} P<0.05,{ }^{*} P<$ $0.01,{ }^{* *} P<0.001$ by 1 -way ANOVA followed by Bonferroni's multiple-comparison test.

recruitment of slanMo from the blood flow, we set up a flow chamber assay system, in which slanMo were perfused over surface-bound, immobilized ICs. In this system, we found a highly efficient capture of slanMo by immobilized ICs under shear stress. By contrast, we could not find significant capture of slanMo in flow chamber slides treated with PBS or a protein loading control. The surface-bound monovalent antibody component of the ICs also mediated some attachment; the capture by ICs was, however, significantly higher, which is in line with the high affinity of slanMo for ICs (31). Surface-bound, immobilized human IgG-ICs (Supplemental Figure 3), but not IgA (Supplemental Figure 4) mediated the recruitment of slanMo from the circulation. These data demonstrate that the interaction of circulating slanMo with immobilized ICs can mediate the local recruitment and firm adhesion of slanMo (Figure 3, A and B). In vitro, the capture of slanMo by ICs immobilized on plastic slides occurred most efficiently at $0.5-1$ dyn/ $\mathrm{cm}^{2}$ surface shear stress (Figure 3C).

IC-induced recruitment of slanMo is mediated by CD16. To compare the capacity of IC-mediated recruitment of slanMo with that of other peripheral blood mononuclear cells (PBMCs), we performed additional flow chamber experiments with purified CD1c ${ }^{+}$DCs, plasmacytoid DCs (pDCs), or T cells. As expected, we did not observe recruitment of T cells, which do not express IC receptors (Figure 3D). However, we also did not observe any recruitment of CD1 $\mathrm{c}^{+} \mathrm{DCs}$ and $\mathrm{pDCs}$, despite a high expression of CD32 by CD1c $\mathrm{c}^{+} \mathrm{DCs}$. In line with this, selective blocking of CD16 on slanMo almost completely abrogated their capture by immobilized ICs, whereas selective blocking of CD32 had no effect. Similarly, the lack of recruitment of mature slanMo, which had downregulated CD16 but maintained CD32 expression (31), independently shows that 
A

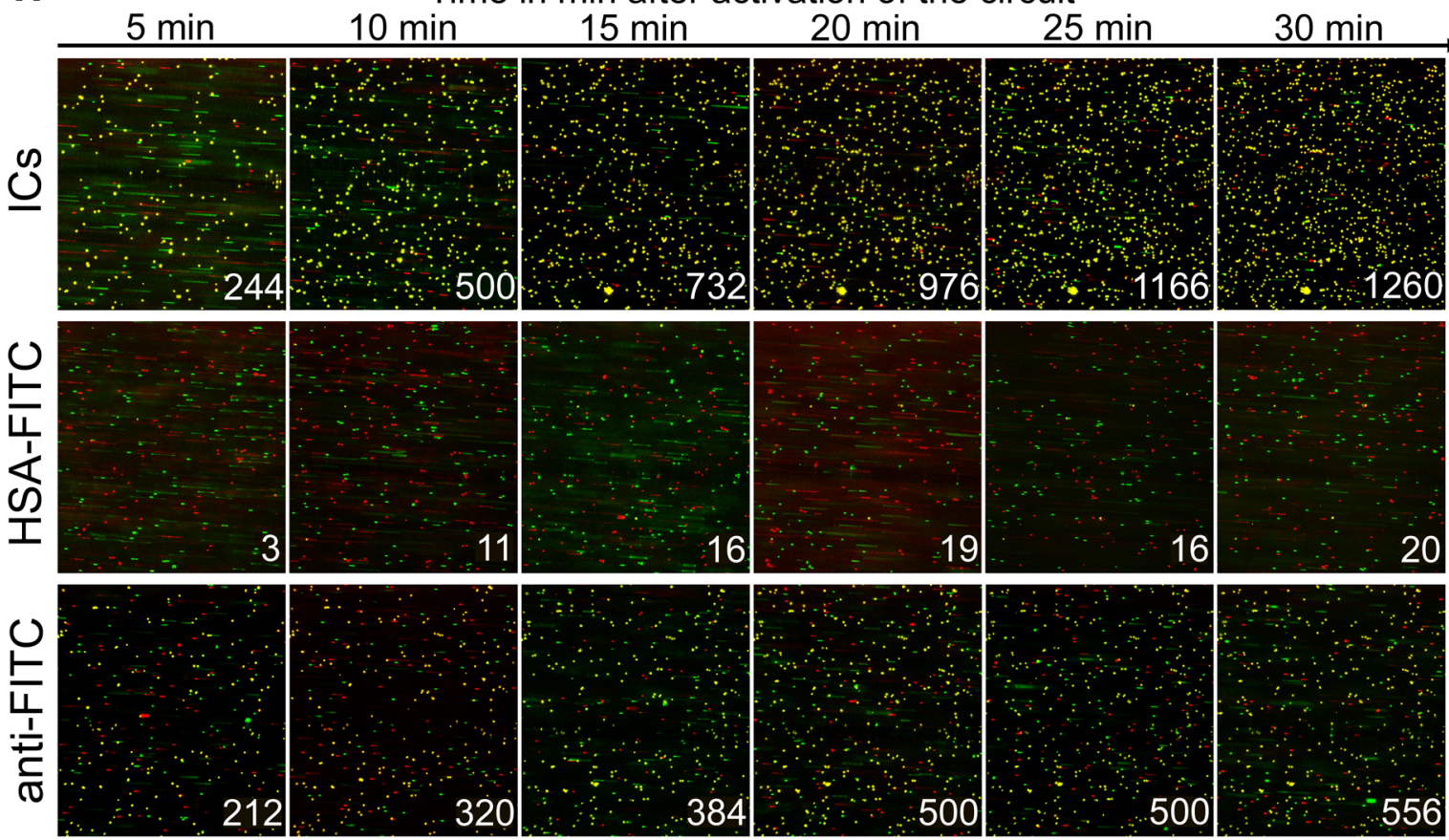

B

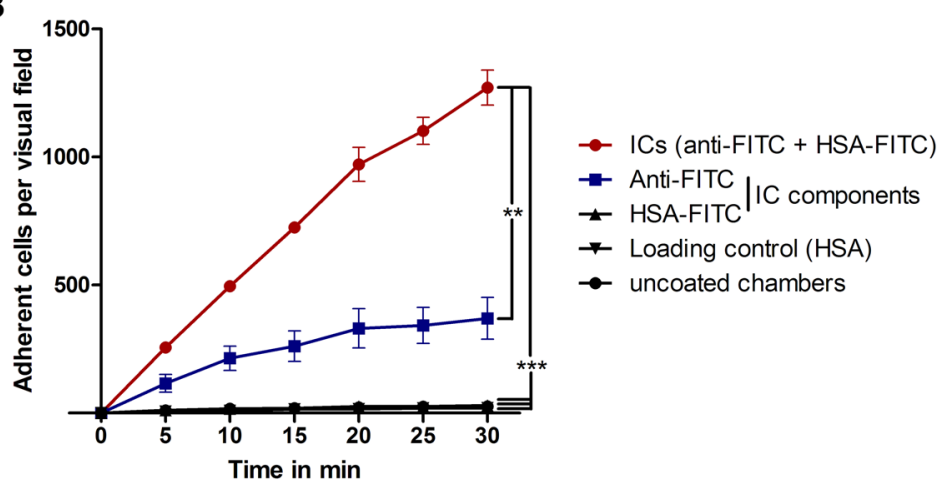

C

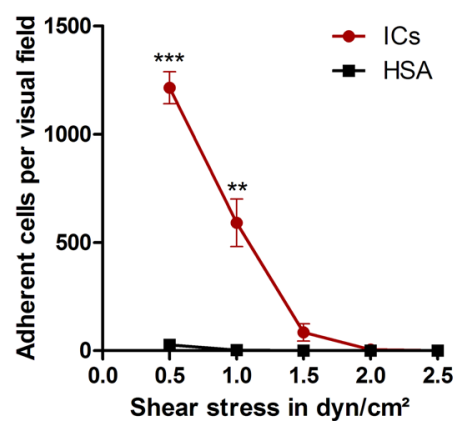

D $\quad \mathrm{CD} 1 \mathrm{c}^{+} \mathrm{DCs}$

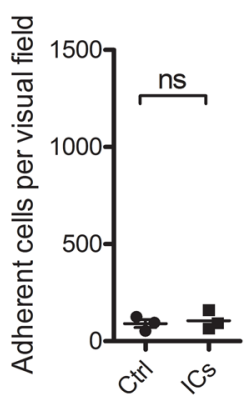

pDCs

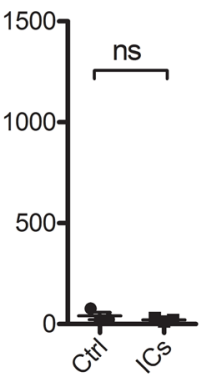

T cells

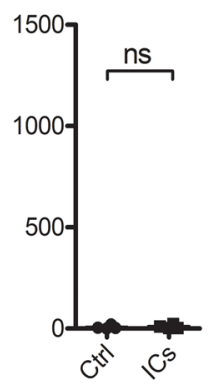

Figure 3. Immobilized ICs recruit slanMo from shear flow conditions. (A and B) Capture of slanMo by immobilized immune complexes (ICs) from the flow. Freshly isolated slanMo were labeled for fluorescence microscopic detection and subsequently perfused over immobilized ICs for 30 minutes at a shear stress of $0.5 \mathrm{dyn} / \mathrm{cm}^{2}$ in a flow chamber assay. Adherent cells appear in yellow after merge of consecutive frames (slanMo in red or green) at intervals of 5 frames in the off-line analysis at the respective time points after the initiation of shear flow. The images are a representative example of 4 experiments. Original magnification, $\times 20$. ICs were preformed by incubating FITC-labeled human serum albumin (HSA, $1 \mathrm{mg} / \mathrm{ml})$ (HSA-FITC) with polyclonal rabbit anti-FITC IgC $(1 \mathrm{mg} / \mathrm{ml})$ (anti-FITC) at 1:6 for 1 hour at $4^{\circ} \mathrm{C}$. (C) Capture of slanMo at increasing levels of surface sheer stress $(n=3)$.

(D) Capture of purified CD1C DCs, pDCs, and CD4+ T cells by immobilized ICs under the same conditions. $n=3$ for CD1c DCs and pDCs and $n=4$ for $\mathrm{CD}^{+} \mathrm{T}$ cells. Mean values are shown $\pm \mathrm{SEM}$. ${ }^{* *} P<0.01,{ }^{* *} P<0.001$ by 1 -way ANOVA followed by Tukey's post hoc test. 
A

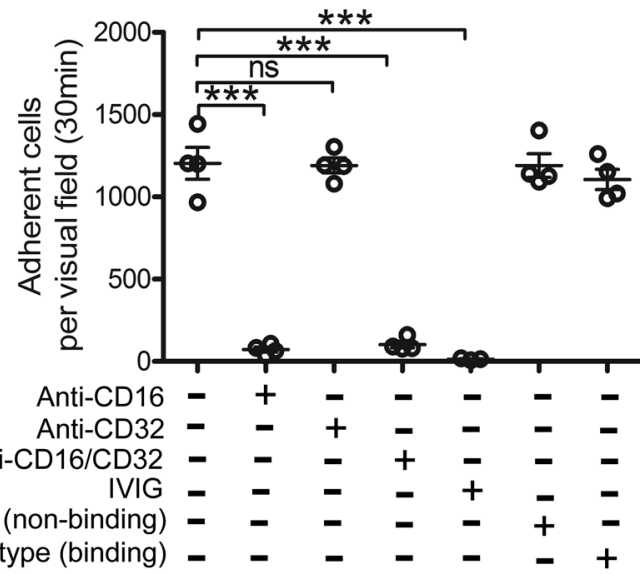

B

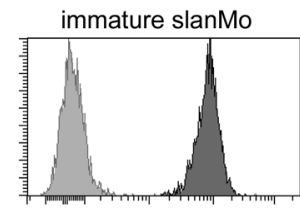

mature slanMo
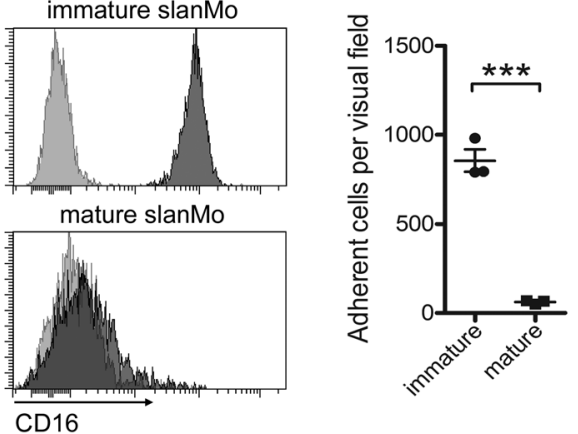

C

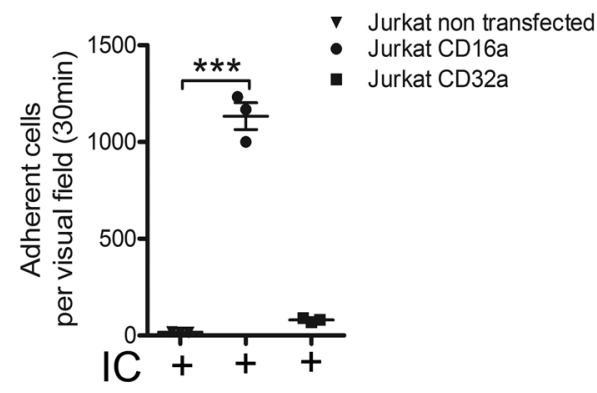

Figure 4. Recruitment of slanMo from the flow requires expression of CD16. (A) CD16-specific capture of slanMo by immobilized immune complexes (ICs). Freshly isolated slanMo were labeled for fluorescence microscopic detection and incubated with specific blocking mAbs for CD16 (3G8), CD32 (AT10), or both as well as a binding (DX17) and a nonbinding (MOPC-21) isotype control. Additionally, intravenous immunoglobulin (IVIG) was used for blocking Fc $\gamma R$. SlanMo subsequently were perfused over immobilized ICs for 30 minutes with an applied surface shear stress of $0.5 \mathrm{dyn} / \mathrm{cm}^{2}(n=4)$. (B) Freshly isolated and CD16-negative mature slanMo of the same donor run over immobilized ICs for 30 minutes at a shear stress of $0.5 \mathrm{dyn} / \mathrm{cm}^{2}(n=3)$. The histograms give an example of the CD16 expression of freshly isolated and mature slanMo of the same donor. (C) CD16a-transfected, CD32a-transfected, and nontransfected Jurkat cells were fluorescently labeled and subsequently run over immobilized ICs for 30 minutes at a shear stress of 0.5 dyn/ $\mathrm{cm}^{2}(n=3)$. Mean values are shown \pm SEM. ${ }^{* * *} P<0.001$. ns, not significant by 1 -way ANOVA followed by Tukey's post hoc test.

IC-mediated capture of slanMo depends on CD16 (Figure 4, A and B). These data were additionally corroborated by using CD16- or CD32-transfected Jurkat cells. In these experiments, CD16-transfected Jurkat cells were efficiently captured by immobilized ICs, while CD32-transfected Jurkat cells were not (Figure 4C). In summary, these studies demonstrate a highly selective role of CD16 for the IC-mediated recruitment of slanMo from the circulation under flow conditions.

CD16-dependent recruitment of slanMo from the flow to antibodies deposited on endothelial cells. To extend these experiments, we investigated whether slanMo can be recruited to endothelial cells by immobilized ICs. We targeted a monolayer of human dermal microvascular endothelial cells (HDMECs) that was grown in flow chamber slides with an antibody specific for endoglin (CD105) (Supplemental Figure 5). Treatment of HDMECs immediately before the perfusion assay with the antibody led to a strong recruitment of slanMo to the endothelial layer compared with untreated HDMECs. This recruitment was nearly completely inhibited by blocking CD16 on slanMo, showing that the capture of slanMo was not due to activation of endothelial cells. Again, capture of slanMo was independent of CD32, as its blocking did not change the number of adherent slanMo (Figure 5).

IgG- and CD16-dependent activation of slanMo to induce an inflammatory immune response. TNF- $\alpha$ is an important orchestrator of inflammatory diseases. It is overexpressed in lupus nephritis and anti-TNF- $\alpha$ therapies proved effective in reducing disease activity (32). Under inflammatory conditions, slanMo are characterized by their high-level TNF- $\alpha$ production. Staining of kidney tissues from class III lupus nephritis for TNF- $\alpha$ expression and slanMo demonstrated the local TNF- $\alpha$ production by slanMo (Figure 6A). Given that slanMo were captured from the flow by a CD16-mediated interaction with ICs, we next determined whether binding of IgG to CD16 induces the production of proinflammatory cytokines. We used an assay in which slanMo were cultured in the presence of surface-bound heat-aggregated IgG. Studying supernatants from these cultures revealed that heat-aggregated IgG induced production of TNF- $\alpha$, IL-6, and CXCL2. Inhibition of the Fc $\gamma$ R signaling pathway using the Syk inhibitor BAY-61-3606 or blocking antibodies for CD16 (clone 3G8) inhibited the proinflammatory feature of slanMo in response to heat-aggregated IgG (Figure 6B). Importantly, a side-byside comparison of slanMo with $\mathrm{CD} 1 \mathrm{c}^{+} \mathrm{DCs}$ and $\mathrm{CD} 14^{+}$monocytes revealed that only slanMo responded to stimulation with heat-aggregated IgG with a strong production of TNF- $\alpha$ and IL-6 (Figure 6C).

One of the local effects of TNF- $\alpha$ is the activation of endothelial cells followed by production of chemokines including fractalkine. Fractalkine was reported to be upregulated in lupus nephritis (11) and is known to stimulate recruitment of CX3CR1 $1^{+} \mathrm{T}$ cells (33) and slanMo. To gain insight into the potential interplay between slanMo and endothelial cells, monolayers of HDMECs were treated with supernatants from IC-stimulated slanMo. As shown in Figure 6D, supernatants of IC-stimulated slanMo induced a strong production of fractalkine among endothelial cells. Neutralizing TNF- $\alpha$ with a TNF- $\alpha$-specific antibody (adalimumab) strongly inhibited secretion of fractalkine (Figure 6D). Collectively, our studies demonstrate 

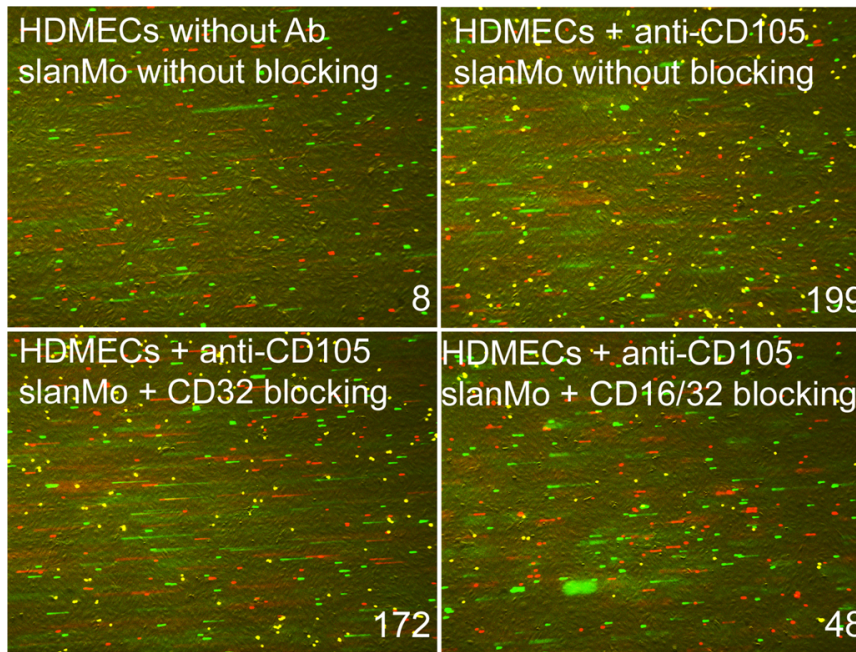
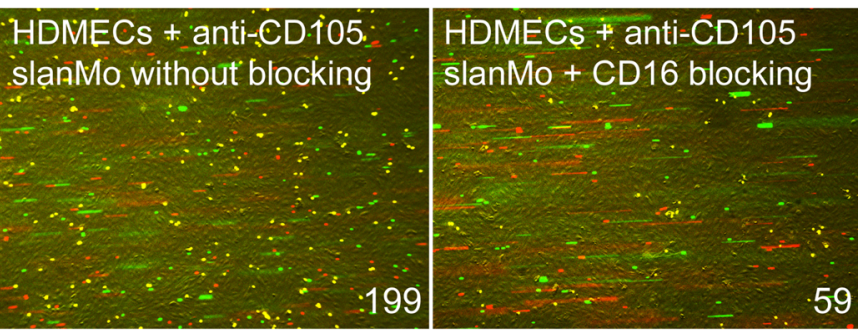

HDMECs + anti-CD105

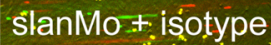

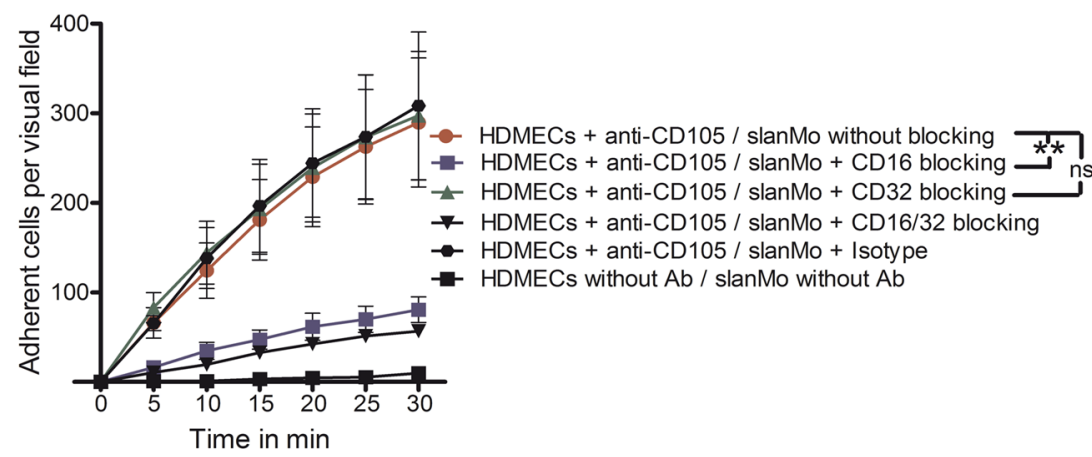

Figure 5. SlanMo are captured by antibodies deposited on endothelial cells. Antibody-dependent capture of slanMo on endothelial cells under shear-flow conditions. Human microvascular endothelial cells (HDMECs) were seeded into flow chamber slides and grown to 100\% confluence. Immediately before the perfusion assay, HDMECs were treated with an antibody against CD105. Subsequently, slanMo that were either untreated or treated with specific blocking mAbs for CD16 (3G8), CD32 (AT10), or both as well as an isotype control (MOPC-21) were perfused for 30 minutes at a shear stress of 0.5 dyn/cm² over the HDMECs. Adherent cells appear in yellow after merge of consecutive frames (slanMo in red or green) at intervals of 5 frames in the off-line analysis at the respective time points after the initiation of shear flow $(n=3)$. Original magnification, $\times 32 .{ }^{*} P<0.01$. ns, not significant by 1 -way ANOVA followed by Tukey's post hoc test.

that IC-mediated cross-linking of CD16 (FcyRIIIa) on slanMo is critical for their local accumulation in glomeruli and for stimulating an inflammatory immune response.

\section{Discussion}

This study identifies slanMo as an early proinflammatory cell type in human lupus nephritis with endocapillary hypercellularity. In particular, we provide evidence for a direct IC- and Fc $\gamma$ R-dependent recruitment of slanMo from the blood circulation to the vascular interface of glomerular capillaries and the subsequent induction of an inflammatory response.

SlanMo are proinflammatory $\mathrm{CD} 16^{+}$monocytes best identified in tissues by their specific expression of the slan marker. In the peripheral blood slanMo were previously shown to specialize in handling IgG-ICs, which is mediated by coexpression of the IC receptors CD16 and CD32 (31). The very efficient binding of ICs is reportedly a unique feature of slanMo when compared with other monocytes or DCs. Uptake of antigen via Fc $\gamma$ Rs strongly enhances the stimulation of antigen-specific $\mathrm{T}$ cells by slanMo (31). In another study, the coexpression of CD16A and CD32A was responsible for potent antibody-dependent cell-mediated cytotoxicity (ADCC) mediated by slanMo (34).

Glomerular monocytes have been reported in human lupus nephritis type III and IV, and their presence was associated with poor prognosis when found at repeated biopsies (11, 35-37). In line with this, mouse models of lupus nephritis demonstrated a proinflammatory role for monocytes activated by ICs $(21,38)$. However, the exact definition of these cells, their recruitment, and their role in the pathogenesis of human lupus nephritis remained unresolved. Our profiling of tissues from patients with lupus nephritis 
A

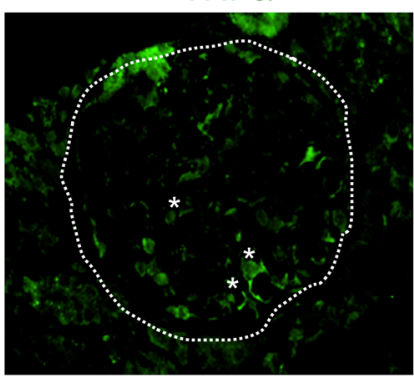

slanMo

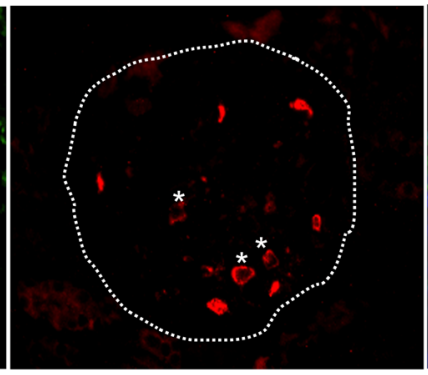

combined

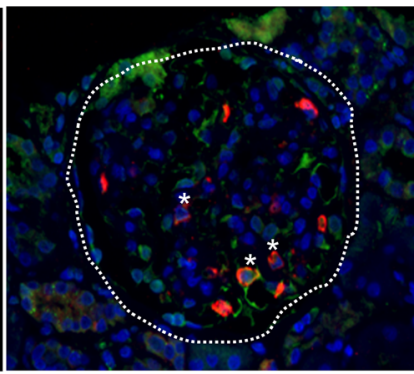

\section{B}

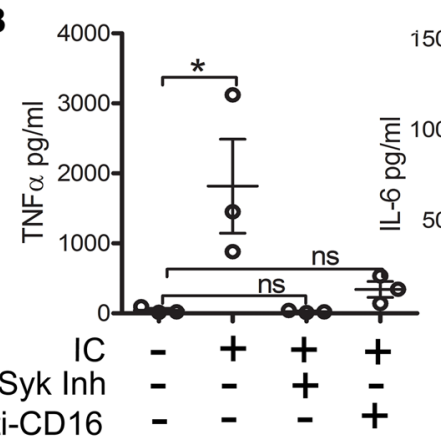

Anti-CD16
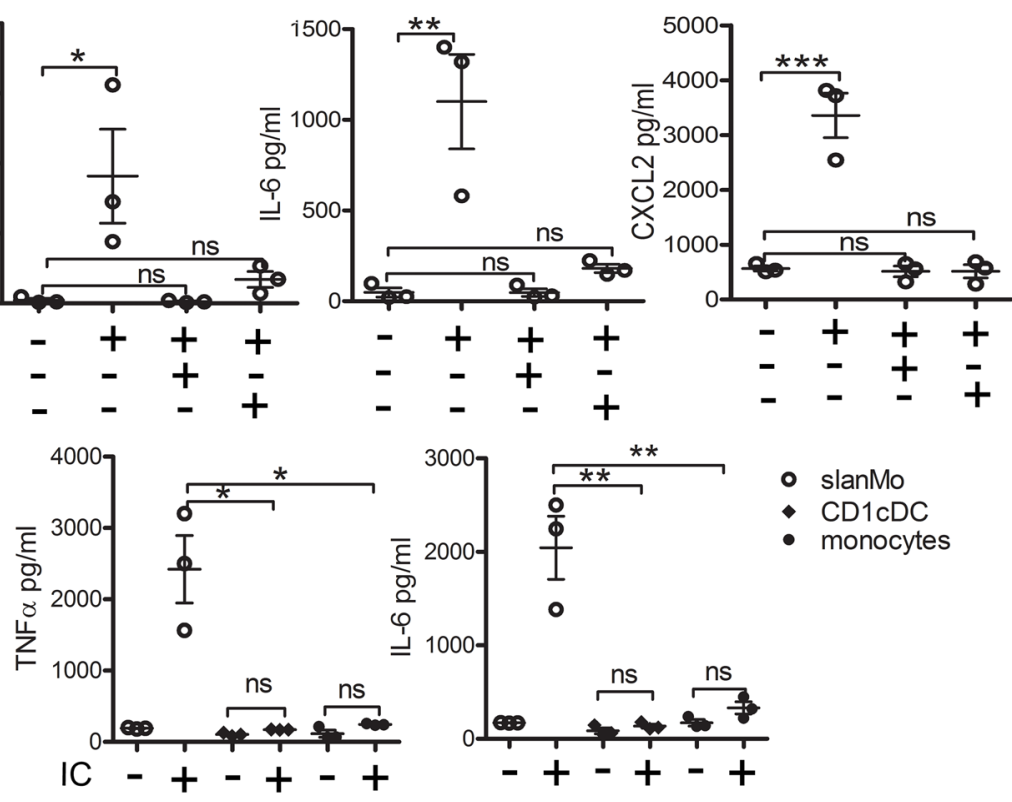

C

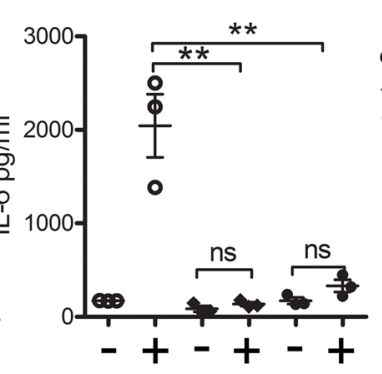

D

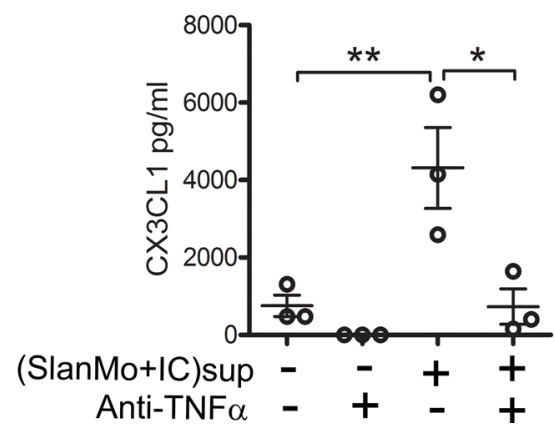

Figure 6. IC-slanMo interaction mediates inflammatory responses. (A) Single-color images and combined colors for the detection of TNF- $\alpha$ (green), slanMo (red), and cell nuclei (DAPI; blue) in lupus nephritis glomeruli class III. Original magnification, $\times 20$. Results are representative of multiple sections from 3 independent donors $(n=3)$. Asterisks indicate double-positive cells. (B) Cytokine induction by immobilized immune complexes (ICs). Freshly isolated slanMo were treated or not with Syk inhibitor or specific blocking mAbs for CD16 (3G8) and incubated with immobilized ICs for 20 hours. TNF- $\alpha$, IL-6, and CXCL2 protein levels in the culture medium were determined by ELISA $(n=3)$. (C) TNF- $\alpha$ and IL-6 levels in the culture medium of CD1c DCs and monocytes in the presence or absence of immobilized ICs $(n=3)$. (D) HDMECs were cultured for 20 hours with supernatants from slanMo previously incubated with immobilized ICs and in the presence or absence of a neutralizing TNF- $\alpha \mathrm{mAb}(10 \mu \mathrm{g} / \mathrm{ml})$. CX3CL1 protein levels were determined by ELISA ( $n=3)$. Mean values are shown $\pm \mathrm{SEM} .{ }^{*} P<0.05,{ }^{* *} P<0.01,{ }^{* *} P<0.001$ by 1 -way ANOVA followed by Bonferroni's multiple-comparison test.

class III identified among all monocytes the preferential presence of slanMo, localized in the glomeruli of these patients. Among the different classes of lupus nephritis, class III and IV are characterized by intraluminal immune aggregates and subendothelial IC deposits in the glomeruli presumably accessible to leukocytes within the blood circulation through the fenestrated endothelium (26). Class III lupus nephritis with involvement of less than $50 \%$ of glomeruli may progress to class IV lupus nephritis with equal and 
more than $50 \%$ of glomeruli being involved (4). Interestingly, in the more advanced cases with class IV lupus nephritis, $\mathrm{CD}^{+} 8^{+}$mononuclear cells outnumbered slanMo. This finding supports a role for slanMo being the first mononuclear phagocytes recruited by ICs in glomeruli, and which then may orchestrate an inflammatory response potentially attracting other cell subsets.

SlanMo were absent from healthy kidneys and from samples where ICs were deposited in the mesangial space (lupus nephritis class I and class II). We here provide strong evidence for the possibility of a direct glomerular recruitment of slanMo from the blood flow by immobilized ICs. Alternatively, as slanMo are a population of nonclassical monocytes for which intravascular patrolling was reported (20), a prolonged IC-mediated retention of already patrolling monocytes as reported previously by Finsterbusch et al. (23) may alternatively account for the increased number of slanMo found in lupus nephritis tissue samples.

When studying the outcome of ICs interacting with slanMo we observed a cytokine and chemokine production relevant for orchestrating a local inflammatory response. Other blood cells that express CD32 but not CD16, like CD1c ${ }^{+}$DCs and pDCs, were not recruited by immobilized ICs. All human IgG subclasses were found to contribute to the capture of slanMo from the flow in their complexed form. However, the capture of slanMo by monomeric IgG3 was considerably higher than the other human IgG subclasses (Supplemental Figure 6). One study described the fucosylation of the Fc tail of IgG3 to be responsible for the enhanced binding affinity of IgG3 for CD16a (39). Although it has been shown that monomeric IgG3 binds more efficiently than monomeric IgG1 to CD32a, CD16a, and CD16b (40), the structural determinants responsible for the differences in IgG binding of individual IgG isotypes remain unknown.

We identified CD16A as the chief surface structure facilitating the IC-mediated capture of slanMo from the flow, and we also observed a more efficient binding of soluble ICs by CD16 versus CD32 in a previous study (31). This difference may be explained by the higher affinity of CD16A versus CD32A for IgG: IgG1-affinity of $1.5 \times 10^{-6} \mathrm{Kd}[\mathrm{M}]$ for CD32A and $4.20 \times 10^{-7} \mathrm{Kd}[\mathrm{M}]$ for CD16 (41). Furthermore, slanMo showed a higher CD16A cell surface expression compared with CD32A (31). An additional level of heterogeneity among CD16 and CD32 is introduced by genetic polymorphisms of these Fc $\gamma$ Rs (42). CD32A has 2 allotypic variants at position 131 named low-responder (H131) and high-responder (R131) (43), whereas F158 and V158 are the allotypic variants described for CD16A. Among these 2 variants, V158 has a greater affinity for IgG3 and the following relative affinities: IgG3 > IgG1 > IgG4 > IgG2 (44).

The capture of slanMo by ICs in vitro was completely inhibited by intravenous IVIGs. These immunoglobulin preparations contain small ICs with the ability to bind and apparently also to block activating Fc $\gamma$ Rs. IVIGs are considered an important option for the treatment of patients with different autoimmune diseases and are recommended for the treatment of refractory cases of lupus nephritis (45). Hence, our findings provide arguments for therapeutic targeting of CD16 for the inhibition of IC-induced recruitment and activation of slanMo.

It was previously shown that the interaction of circulating neutrophils with ICs deposited in the vascular bed was largely dependent on GPI-linked CD16B and in some models on both CD16B and CD32A (46-48). Although the presence of neutrophils is not regarded as a classic feature of IC-induced lupus nephritis, they contribute to the systemic inflammatory response in lupus (49), and we previously described neutrophil extracellular traps in anti-neutrophil cytoplasmic autoantibody-induced small vessel vasculitis glomerulonephritis (50). Interestingly, there is evidence for neutrophils enhancing the survival and proinflammatory properties of slanMo (51-53).

We demonstrated that IC binding by slanMo induces inflammatory downstream signaling after cross-linking of Fc $\gamma$ Rs. CD16 engagement by immobilized ICs resulted in the secretion of TNF- $\alpha$ and IL- 6 as well as the neutrophil-recruiting chemokine CXCL2 by slanMo. TNF- $\alpha$ production by slanMo stimulated endothelial cells to produce fractalkine, potentially initiating and maintaining a circuit of local tissue inflammation and cell retention.

These findings fit very well with studies by Ahn et al. describing the TNF- $\alpha$-dependent activation of endothelial cells to produce fractalkine (54). Furthermore, fractalkine and CX3CR1 were reported to mediate leukocyte capture, firm adhesion, and activation under physiologic flow (55). This mechanism has also been studied in the context of human renal inflammatory states (56), and both fractalkine and CX3CR1 were shown to play key roles in the patrolling of mouse monocytes in glomeruli (23), their migration (57), and transmigration of endothelial cells (57-59). Antagonizing fractalkine in lupus-prone $\mathrm{MRL} / \mathrm{lpr}$ mice delays the generation and ameliorates the progression of lupus nephritis (60). Fractalkine has been shown to contribute to the development of leukocytoclastic vasculitis, a type III hypersensitivity reaction (Arthus reaction) induced by ICs mainly by regulating neutrophil and mast cell recruitment and 
cytokine expression (61). Similarly, mice lacking CX3CR1 showed a reduced IC-induced edema and hemorrhage during the Arthus reactions. This was correlated with reduced neutrophil and mast cell accumulation in the skin and peritoneum as well as with the increased expression of the proinflammatory cytokines TNF- $\alpha$ and IL-6 (61). Activation of mast cells, neutrophils, and monocytes induces secretion of TNF- $\alpha$, which is known to induce expression of fractalkine in endothelial cells (54) and in turn could enhance migration of other CX3CR1-positive cells.

The finding of a local production of TNF- $\alpha$ by slanMo in lupus nephritis documents their direct contribution to the local inflammatory response. SlanMo have been described as a cell type producing large amounts of TNF- $\alpha$ after stimulation with TLR7/8 as well as TLR4 ligands (24).

Anti-TNF- $\alpha$ treatment proved effective in the reduction of lupus nephritis in patients as well as mouse models, thereby providing strong evidence for an important role for TNF- $\alpha$ in stimulating the local inflammatory process in lupus nephritis. However, the therapeutic value of targeting TNF- $\alpha$ in lupus nephritis is limited, as increased rates of infections were noticed in anti-TNF- $\alpha$-treated patients (62) and covers only one aspect of the complex inflammatory reaction.

The IC-mediated recruitment and activation of slanMo presented in this study may also apply to other kidney diseases. For example, in acute antibody-mediated kidney-allograft rejection, infiltrating macrophages are predictors for graft loss (63). Moreover, the findings presented in this study may have direct relevance for setting off a beneficial antiviral immune response at vascular interfaces in already immunized and seroconverted patients. Here, IgG-opsonized viral particles may be sensed at the vascular interface by slanMo, inducing a subsequent proinflammatory response to control viral spread. However, the same mechanisms may account for the induction of inflammatory vascular complications in patients with herpes zoster, such as transient ischemic attacks, stroke, aneurysm, sinus thrombosis, giant cell arteritis, and granulomatous aortitis (64).

$\mathrm{CD} 6^{+}$monocytes are a heterogeneous cell population, as precisely revealed by recent transcriptomic and phenotypic studies (65-66). We are just beginning to understand their role in immunity. It currently appears as if the phenotype and function of $\mathrm{CD} 16^{+}$monocytes presents as a spectrum, with IL-10-producing, nonpatrolling (20), intermediate CD16 ${ }^{+}$monocytes (high expression of CD14, CD11b, CD163, and CD36) and with low-level IL-10-producing, patrolling, nonclassical monocytes (low or absent expression of CD14, CD11b, CD163, and CD36) (20). The side-by-side comparison of slanMo with other CD16 monocytes demonstrated a particularly high capacity for slanMo to produce IL-12 (13) and TNF- $\alpha$ (67). In line with this, it was demonstrated that slanMo have a transcriptomic signature distinct from slan-negative $\mathrm{CD}_{16}{ }^{+}$monocytes (19). CD16 ${ }^{+}$intermediate monocytes as well as nonclassical monocytes can develop from blood monocytes (68); whether this also applies to slanMo or whether they develop from a separate precursor cell requires further studies.

Once slanMo are recruited to tissues, different fates may ensue. SlanMo were shown in vitro to differentiate into proinflammatory DCs $(13,15,69)$ or macrophages (14) depending on the local microenvironment. Similarly, in vivo slanMo were identified as IL-23-producing inflammatory dermal DCs in psoriasis (16), as DCs in tonsils (14), or as immature IL-10-producing macrophage-like cells in renal cell carcinoma (70).

In summary, lupus nephritis is a major cause of acute and chronic kidney disease in women frequently leading to end-stage renal disease and limited treatment options. Our study adds to the understanding of the mechanisms involved in the initiation and perpetuation of IC-mediated inflammation and provides arguments for the design of slanMo-specific innovative therapeutic interventions in ICmediated inflammatory diseases (71).

\section{Methods}

Patient samples. Renal biopsies were categorized into class I, II, III, IV, and V of lupus nephritis according to the ISN/RPS classification and based on light microscopy, electron microscopy, and immunohistology with staining for immunoglobulins, complement, and fibrin/fibrinogen.

Cell separation and flow cytometry. PBMCs of healthy donors were isolated from fresh buffy coats by density-gradient centrifugation over Ficoll (Biochrom $\mathrm{GmbH}$ ). Purified leukocyte populations were obtained from PBMCs by magnetic cell separation using the autoMACS Pro Separator (Miltenyi Biotec). SlanMo were isolated as described previously (15). Purity of cell types after magnetic cell separation was determined by staining with specific markers and quantification by flow cytometry using a FACSCanto (BD Biosciences). 
Solid-phase IC assay. Heat-aggregated human IgG $(100 \mu \mathrm{g} / \mathrm{ml})$ was directly bound to tissue culture plates (Corning) in sterile PBS overnight at $4^{\circ} \mathrm{C}$. Plates were then washed with sterile PBS and freshly isolated human leukocyte populations were added and left to stand for 20 hours in a $37^{\circ} \mathrm{C}$ cell incubator with $5 \% \mathrm{CO}_{2}$. In specified experiments, slanMo were incubated with $25 \mu \mathrm{g} / \mathrm{ml}$ anti-human CD16 antibody (3G8) (BioLegend) or $4 \mu \mathrm{M}$ Syk inhibitor BAY-61-3606 (Enzo Life Sciences). After 20 hours the cell culture medium was tested by ELISA for IC-induced cytokine/chemokine responses: IL- 6 , TNF- $\alpha$ (both from BD Pharmingen), and CXCL2 (R\&D Systems).

Flow chamber assay. ICs and respective controls were immobilized by incubating $\mu$-slides I0.8 Luer (IBIDI) for at least 6 hours at $4^{\circ} \mathrm{C}$ with either $200 \mu 1$ of $50 \mu \mathrm{g} / \mathrm{ml}$ ICs or appropriate concentrations of controls in PBS. Endothelial cell monolayers were grown in flow chamber slides as described above. Purified cells were labeled with $15 \mu \mathrm{mol} / 1$ 5-chloromethylfluorescein diacetate (CMFDA) (Life Technologies) in serum-free medium (X-VIVO 15, Lonza) for 45 minutes at $4^{\circ} \mathrm{C}$ followed by washing with medium. For analysis of receptor-specific adhesion, CMFDA-labeled slanMo were incubated with 50 $\mu \mathrm{g} / \mathrm{ml}$ blocking antibodies or $25 \mathrm{mg}$ of IVIG for 30 minutes at $4^{\circ} \mathrm{C}$. Flow chamber slides were continuously perfused with $1 \times 10^{6}$ cells per sample in $5 \mathrm{ml}$ conditioned medium at ambient temperature. Every sample was run for 30 minutes in an equilibrated bubble-free system that was driven by a peristaltic pump (ISM 795C, Ismatec). The surface shear stress within the flow chamber slides was adjusted to $0.5 \mathrm{dyn} / \mathrm{cm}^{2}$ unless otherwise stated. The number of adherent cells per visible field was determined by video microscopy using the Leica Z16-APO fluorescence microscope (Leica), the Coolsnap EZ digital camera (Photometrics), and Metavue software (Molecular Devices). Images were recorded and attached cells were quantified off-line; adherent cells appear in yellow after merge of consecutive frames (slanMo in red or green) at intervals of 5 frames in the off-line analysis at the respective time points after shear flow was started. For all shown experiments, the counted visual field had the same size and was located in the central part of the chamber slides.

Mouse model. Immunodeficient nonobese diabetic (NOD)-SCID IL-2 $\gamma$ chain receptor (NSG) mice were purchased from The Jackson Laboratory. All animals were housed in the central animal facility of the University of Heidelberg. NSG mice of either sex, between 8 and 10 weeks old, were injected in the lateral tail vein with $5 \mathrm{mg} / \mathrm{ml}$ of preformed IgG-ICs or PBS as control in a $100 \mu \mathrm{l}$ volume. Heataggregated IgGs were prepared as described above. Freshly isolated slanMo $\left(8 \times 10^{6}\right)$ were fluorescently labeled with CFSE (Invitrogen) according to the manufacturer's protocol and injected 7 hours later in the lateral tail vein in a $100 \mu 1$ volume. For blocking of CD16, cells were incubated with 25 $\mu \mathrm{g} / \mathrm{ml}$ anti-human CD16 antibody (3G8) (BD Biosciences) or mouse IgG1 (MOPC-21) (BioLegend) as nonbinding isotype antibody for 30 minutes on ice prior to i.v. injection. Mice were euthanized 2 hours after cell injection and kidneys were collected for further analysis. Control groups received the same dose of ICs, followed by injection of CFSE-labeled CD16a/CD32a-transfected Jurkat or WT Jurkat cells.

Direct immunofluorescence of mouse kidneys. Portions of snap-frozen mouse kidneys embedded in Tissue-Tek OCT Compound (Sakura Finetek) were cryosectioned $(5 \mu \mathrm{m})$, fixed in $4 \%$ paraformaldehyde (PFA) (Thermo Fischer Scientific) for 10 minutes, and blocked with 3\% BSA and 3\% normal goat serum. Kidney glomeruli were identified by staining with rabbit anti-mouse collagen IV antibodies (Progen Biotechnik) and Alexa Fluor 594-conjugated goat anti-rabbit IgG (Invitrogen). Sections were mounted with antifade reagent (Invitrogen) and examined under a Leica DM 5500 B microscope (Leica Microsystems). Glomeruli containing CFSE-labeled cells were counted and expressed as positive glomeruli/total number of glomeruli. At least 25 glomeruli were examined in each tissue section.

Statistics. Statistical analyses were performed using 1-way ANOVA followed by Tukey's post hoc test unless otherwise indicated. Levels of statistical significance were $P<0.05\left({ }^{*}\right), P<0.01\left({ }^{* *}\right)$, and $P<0.001\left({ }^{* *}\right)$. Data are shown as mean \pm SEM. Graphs were prepared and statistics were calculated in Prism 5 (GraphPad Software).

Study approval. All work contained in this publication was approved by the Institutional Review Board of the University of Heidelberg, Germany. Healthy blood donors gave written informed consent in accordance with the ethics principles stated in the Declaration of Helsinki.

All mouse experiments were carried out according to the Guidelines for Animal Welfare of the State of Baden-Württemberg. 


\section{Author contributions}

FO, TD, ASL, SO, and MM performed experiments. TD, ASL, FO, AHE, MS, EFG, HJG, and KS designed research. EFG and HJG provided renal biopsy samples. TD, ASL, FO, and KS analyzed and interpreted data. KS, TD, and FO wrote the manuscript.

\section{Acknowledgments}

The authors thank Gabriele Schmidt (German Cancer Research Center [DKFZ], Heidelberg), Galina Grabe, Silvia Pezer, and Stefan Meisel (Department of Dermatology, Heidelberg University Hospital) for excellent technical assistance. Anke S. Lonsdorf was supported by the Olympia-Morata-Program of the Medical Faculty of the University of Heidelberg. This work was supported in part by grants from the Deutsche Forschungsgemeinschaft (DFG, SCHA 1693/1-1 to K. Schäkel) and SFB TR156 CO2 to K. Schäkel and F. Olaru.

Address correspondence to: Knut Schäkel, Department of Dermatology, University Hospital Heidelberg, Im Neuenheimer Feld 440, 69120 Heidelberg, Germany. Phone: 49.6221.56.8511; Email: Knut.Schaekel@ med.uni-heidelberg.de.

TD's present address is: Dermatology Branch, National Institute of Arthritis and Musculoskeletal and Skin Diseases, NIH, Bethesda, Maryland, USA.

1. Cohen SD, Kimmel PL. Immune complex renal disease and human immunodeficiency virus infection. Semin Nephrol. 2008;28(6):535-544.

2. de Zubiria Salgado A, Herrera-Diaz C. Lupus nephritis: an overview of recent findings. Autoimmune Dis. 2012;2012:849684.

3. Gross WL, Trabandt A, Reinhold-Keller E. Diagnosis and evaluation of vasculitis. Rheumatology (Oxford). 2000;39(3):245-252.

4. Weening JJ, et al. The classification of glomerulonephritis in systemic lupus erythematosus revisited. Kidney Int. 2004;65(2):521-530

5. Means TK, Latz E, Hayashi F, Murali MR, Golenbock DT, Luster AD. Human lupus autoantibody-DNA complexes activate DCs through cooperation of CD32 and TLR9. J Clin Invest. 2005;115(2):407-417.

6. Vollmer J, et al. Immune stimulation mediated by autoantigen binding sites within small nuclear RNAs involves Toll-like receptors 7 and 8. J Exp Med. 2005;202(11):1575-1585.

7. Kurts C, Panzer U, Anders HJ, Rees AJ. The immune system and kidney disease: basic concepts and clinical implications. Nat Rev Immunol. 2013;13(10):738-753.

8. Nimmerjahn F, Ravetch JV. Fcgamma receptors as regulators of immune responses. Nat Rev Immunol. 2008;8(1):34-47.

9. Bergtold A, Gavhane A, D'Agati V, Madaio M, Clynes R. FcR-bearing myeloid cells are responsible for triggering murine lupus nephritis. J Immunol. 2006;177(10):7287-7295.

10. Clynes R, Dumitru C, Ravetch JV. Uncoupling of immune complex formation and kidney damage in autoimmune glomerulonephritis. Science. 1998;279(5353):1052-1054.

11. Yoshimoto S, et al. Elevated levels of fractalkine expression and accumulation of $\mathrm{CD}_{16}{ }^{+}$monocytes in glomeruli of active lupus nephritis. Am J Kidney Dis. 2007;50(1):47-58.

12. Ziegler-Heitbrock L, et al. Nomenclature of monocytes and dendritic cells in blood. Blood. 2010;116(16):e74-e80.

13. Schäkel K, et al. Human 6-sulfo LacNAc-expressing dendritic cells are principal producers of early interleukin-12 and are controlled by erythrocytes. Immunity. 2006;24(6):767-777.

14. Micheletti A, et al. slan/M-DC8 ${ }^{+}$cells constitute a distinct subset of dendritic cells in human tonsils. Oncotarget. 2016;7(1):161-175.

15. Schäkel K, Mayer E, Federle C, Schmitz M, Riethmüller G, Rieber EP. A novel dendritic cell population in human blood: onestep immunomagnetic isolation by a specific mAb (M-DC8) and in vitro priming of cytotoxic T lymphocytes. Eur J Immunol. 1998;28(12):4084-4093.

16. Hänsel A, et al. Human slan (6-sulfo LacNAc) dendritic cells are inflammatory dermal dendritic cells in psoriasis and drive strong TH17/TH1 T-cell responses. J Allergy Clin Immunol. 2011;127(3):787-94.e1.

17. Guenova E, et al. IL-4 abrogates T(H)17 cell-mediated inflammation by selective silencing of IL-23 in antigen-presenting cells Proc Natl Acad Sci USA. 2015;112(7):2163-2168.

18. Van Leeuwen-Kerkhoff N, et al. Transcriptional profiling reveals functional dichotomy between human slan ${ }^{+}$non-classical $^{-}$ monocytes and myeloid dendritic cells. J Leukoc Biol. 2017;102(4):1055-1068.

19. Hofer TP, et al. slan-defined subsets of CD16-positive monocytes: impact of granulomatous inflammation and M-CSF receptor mutation. Blood. 2015;126(24):2601-2610.

20. Cros J, et al. Human CD14dim monocytes patrol and sense nucleic acids and viruses via TLR7 and TLR8 receptors. Immunity. 2010;33(3):375-386.

21. Carlin LM, et al. Nr4a1-dependent Ly6C(low) monocytes monitor endothelial cells and orchestrate their disposal. Cell. 2013;153(2):362-375.

22. Devi S, et al. Multiphoton imaging reveals a new leukocyte recruitment paradigm in the glomerulus. Nat Med. 2013;19(1):107-112

23. Finsterbusch M, et al. Patrolling monocytes promote intravascular neutrophil activation and glomerular injury in the acutely 
inflamed glomerulus. Proc Natl Acad Sci USA. 2016;113(35):E5172-E5181.

24. Hänsel A, et al. Human 6-sulfo LacNAc (slan) dendritic cells have molecular and functional features of an important proinflammatory cell type in lupus erythematosus. J Autoimmun. 2013;40:1-8.

25. Abbate M, et al. Complement-mediated dysfunction of glomerular filtration barrier accelerates progressive renal injury. $J A m$ Soc Nephrol. 2008;19(6):1158-1167.

26. Kashgarian M,Hayslett JP. Renal Involvement in Systemic Lupus Erythematosus. In: Tisher CC, Brenner BM, eds. Renal Pathology with Clinical and Functional Correlations (2nd edition). Philadelphia, USA: Lippincott JB; 1994:442-471.

27. Arbuckle MR, et al. Development of autoantibodies before the clinical onset of systemic lupus erythematosus. $N$ Engl J Med. 2003;349(16):1526-1533.

28. Wang Y, et al. Laser microdissection-based analysis of cytokine balance in the kidneys of patients with lupus nephritis. Clin Exp Immunol. 2010;159(1):1-10.

29. Abrass CK. Mechanisms of immune complex formation and deposition in renal stuctures. In: Neilson EG, Couser WG, eds. Immunologic Renal Disease. Philadelphia, PA: Lippincott Williams \& Wilkins; 2001:277-295.

30. Nourshargh S, Alon R. Leukocyte migration into inflamed tissues. Immunity. 2014;41(5):694-707.

31. Döbel T, et al. Fc $\gamma$ RIII (CD16) equips immature 6-sulfo LacNAc-expressing dendritic cells (slanDCs) with a unique capacity to handle IgG-complexed antigens. Blood. 2013;121(18):3609-3618.

32. Hayat SJ, Uppal SS, Narayanan Nampoory MR, Johny KV, Gupta R, Al-Oun M. Safety and efficacy of infliximab in a patient with active WHO class IV lupus nephritis. Clin Rheumatol. 2007;26(6):973-975.

33. Staumont-Sallé D, et al. $\mathrm{CX}_{3} \mathrm{CL} 1$ (fractalkine) and its receptor $\mathrm{CX}_{3} \mathrm{CR} 1$ regulate atopic dermatitis by controlling effector $\mathrm{T}$ cell retention in inflamed skin. J Exp Med. 2014;211(6):1185-1196.

34. Schmitz M, Zhao S, Schäkel K, Bornhäuser M, Ockert D, Rieber EP. Native human blood dendritic cells as potent effectors in antibody-dependent cellular cytotoxicity. Blood. 2002;100(4):1502-1504.

35. Lech M, Anders HJ. Macrophages and fibrosis: How resident and infiltrating mononuclear phagocytes orchestrate all phases of tissue injury and repair. Biochim Biophys Acta. 2013;1832(7):989-997.

36. Duffield JS. Macrophages in kidney repair and regeneration. J Am Soc Nephrol. 2011;22(2):199-201.

37. Hill GS, et al. Predictive power of the second renal biopsy in lupus nephritis: significance of macrophages. Kidney Int. 2001;59(1):304-316.

38. Santiago-Raber ML, et al. Fcgamma receptor-dependent expansion of a hyperactive monocyte subset in lupus-prone mice. Arthritis Rheum. 2009;60(8):2408-2417.

39. Li H, et al. Optimization of humanized IgGs in glycoengineered Pichia pastoris. Nat Biotechnol. 2006;24(2):210-215.

40. Bruhns $P$, et al. Specificity and affinity of human Fcgamma receptors and their polymorphic variants for human IgG subclasses Blood. 2009;113(16):3716-3725.

41. Bournazos S, Ravetch JV. Fcy receptor pathways during active and passive immunization. Immunol Rev. 2015;268(1):88-103.

42. van Sorge NM, van der Pol WL, van de Winkel JG. FcgammaR polymorphisms: Implications for function, disease susceptibility and immunotherapy. Tissue Antigens. 2003;61(3):189-202.

43. Warmerdam PA, van de Winkel JG, Gosselin EJ, Capel PJ. Molecular basis for a polymorphism of human Fc gamma receptor II (CD32). J Exp Med. 1990;172(1):19-25.

44. Shields RL, et al. High resolution mapping of the binding site on human IgG1 for Fc gamma RI, Fc gamma RII, Fc gamma RIII, and FcRn and design of IgG1 variants with improved binding to the Fc gamma R. J Biol Chem. 2001;276(9):6591-6604.

45. Mok CC. Therapeutic options for resistant lupus nephritis. Semin Arthritis Rheum. 2006;36(2):71-81.

46. Skilbeck CA, Lu X, Sheikh S, Savage CO, Nash GB. Capture of flowing human neutrophils by immobilised immunoglobulin: roles of Fc-receptors CD16 and CD32. Cell Immunol. 2006;241(1):26-31.

47. Coxon A, et al. Fc gamma RIII mediates neutrophil recruitment to immune complexes. a mechanism for neutrophil accumulation in immune-mediated inflammation. Immunity. 2001;14(6):693-704.

48. Pankhurst T, Nash G, Williams J, Colman R, Hussain A, Savage C. Immunoglobulin subclass determines ability of immunoglobulin (Ig)G to capture and activate neutrophils presented as normal human IgG or disease-associated anti-neutrophil cytoplasm antibody (ANCA)-IgG. Clin Exp Immunol. 2011;164(2):218-226.

49. Kienhöfer D, et al. Experimental lupus is aggravated in mouse strains with impaired induction of neutrophil extracellular traps. JCI Insight. 2017;2(10):e92920.

50. Kessenbrock K, et al. Netting neutrophils in autoimmune small-vessel vasculitis. Nat Med. 2009;15(6):623-625.

51. Micheletti A, et al. Neutrophils promote 6-sulfo LacNAc ${ }^{+}$dendritic cell (slanDC) survival. J Leukoc Biol. 2013;94(4):705-710.

52. Costantini $\mathrm{C}$, et al. On the potential involvement of CD11d in co-stimulating the production of interferon- $\gamma$ by natural killer cells upon interaction with neutrophils via intercellular adhesion molecule-3. Haematologica. 2011;96(10):1543-1547.

53. Costantini C, et al. Human neutrophils interact with both 6-sulfo LacNAc ${ }^{+} \mathrm{DC}$ and NK cells to amplify NK-derived IFN $\{$ gamma $\}$ : role of CD18, ICAM-1, and ICAM-3. Blood. 2011;117(5):1677-1686.

54. Ahn SY, et al. Tumor necrosis factor-alpha induces fractalkine expression preferentially in arterial endothelial cells and mithramycin A suppresses TNF-alpha-induced fractalkine expression. Am J Pathol. 2004;164(5):1663-1672.

55. Fong AM, et al. Fractalkine and CX3CR1 mediate a novel mechanism of leukocyte capture, firm adhesion, and activation under physiologic flow. J Exp Med. 1998;188(8):1413-1419.

56. Cockwell P, Chakravorty SJ, Girdlestone J, Savage CO. Fractalkine expression in human renal inflammation. J Pathol. 2002;196(1):85-90.

57. Ancuta P, et al. Fractalkine preferentially mediates arrest and migration of CD16 ${ }^{+}$monocytes. J Exp Med. 2003;197(12):1701-1707.

58. Ancuta P, Moses A, Gabuzda D. Transendothelial migration of CD16 $6^{+}$monocytes in response to fractalkine under constitutive and inflammatory conditions. Immunobiology. 2004;209(1-2):11-20.

59. Ancuta P, Wang J, Gabuzda D. CD16 ${ }^{+}$monocytes produce IL-6, CCL2, and matrix metalloproteinase-9 upon interaction with CX3CL1-expressing endothelial cells. J Leukoc Biol. 2006;80(5):1156-1164.

60. Inoue A, et al. Antagonist of fractalkine (CX3CL1) delays the initiation and ameliorates the progression of lupus nephritis in MRL/lpr mice. Arthritis Rheum. 2005;52(5):1522-1533. 
61. Morimura S, Sugaya M, Sato S. Interaction between CX3CL1 and CX3CR1 regulates vasculitis induced by immune complex deposition. Am J Pathol. 2013;182(5):1640-1647.

62. Aringer M, Smolen JS. The role of tumor necrosis factor-alpha in systemic lupus erythematosus. Arthritis Res Ther. 2008;10(1):202.

63. Colvin RB. Antibody-mediated renal allograft rejection: diagnosis and pathogenesis. J Am Soc Nephrol. 2007;18(4):1046-1056.

64. Chiang F, Panyaping T, Tedesqui G, Sossa D, Costa Leite C, Castillo M. Varicella zoster CNS vascular complications. A report of four cases and literature review. Neuroradiol J. 2014;27(3):327-333.

65. Villani AC, et al. Single-cell RNA-seq reveals new types of human blood dendritic cells, monocytes, and progenitors. Science. 2017;356(6335):eaah4573.

66. Thomas GD, et al. Human blood monocyte subsets: A new gating strategy defined using cell surface markers identified by mass cytometry. Arterioscler Thromb Vasc Biol. 2017;37(8):1548-1558.

67. Dutertre CA, et al. Pivotal role of $\mathrm{M}-\mathrm{DC} 8^{+}$monocytes from viremic HIV-infected patients in TNF $\alpha$ overproduction in response to microbial products. Blood. 2012;120(11):2259-2268.

68. Patel AA, et al. The fate and lifespan of human monocyte subsets in steady state and systemic inflammation. J Exp Med 2017;214(7):1913-1923

69. Randolph GJ, Sanchez-Schmitz G, Liebman RM, Schäkel K. The CD16(+) (FcgammaRIII(+)) subset of human monocytes preferentially becomes migratory dendritic cells in a model tissue setting. J Exp Med. 2002;196(4):517-527.

70. Toma M, et al. Accumulation of tolerogenic human 6-sulfo LacNAc dendritic cells in renal cell carcinoma is associated with poor prognosis. Oncoimmunology. 2015;4(6):e1008342.

71. Bippes CC, et al. A novel modular antigen delivery system for immuno targeting of human 6-sulfo LacNAc-positive blood dendritic cells (SlanDCs). PLoS One. 2011;6(1):e16315 